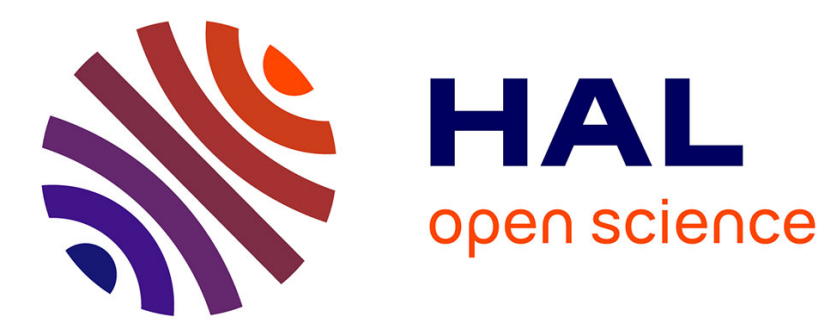

\title{
L'ombre de l'enfant non né sur la grossesse suivante Claire Squires
}

\section{To cite this version:}

Claire Squires. L'ombre de l'enfant non né sur la grossesse suivante: Mort in utero; deuil périnatal;enfant de remplacement; mélancolie;hypocondrie. Cliniques méditerranéennes, 2004, 69, pp.269 288. $10.3917 / \mathrm{cm} .069 .0269$. hal-01451245

\section{HAL Id: hal-01451245 \\ https://hal.science/hal-01451245}

Submitted on 31 Jan 2017

HAL is a multi-disciplinary open access archive for the deposit and dissemination of scientific research documents, whether they are published or not. The documents may come from teaching and research institutions in France or abroad, or from public or private research centers.
L'archive ouverte pluridisciplinaire HAL, est destinée au dépôt et à la diffusion de documents scientifiques de niveau recherche, publiés ou non, émanant des établissements d'enseignement et de recherche français ou étrangers, des laboratoires publics ou privés. 


\title{
L'OMBRE DE L'ENFANT NON Né SUR LA GROSSESSE SUIVANTE
}

\author{
Claire Squires
}

ERES | Cliniques méditerranéennes

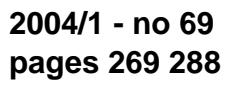

ISSN 0762-7491

Article disponible en ligne l'adresse:

http://www.cairn.info/revue-cliniques-mediterraneennes-2004-1-page-269.htm

Pour citer cet article :

Squires Claire, L'ombre de l'enfant non né sur la grossesse suivante,

Cliniques méditerranéennes, 2004/1 no 69, p. 269-288. DOI : 10.3917/cm.069.0269

Distribution ectronique Cairn.info pour ERES.

ERES. Tous droits rerv pour tous pays.

La reproduction ou reprentation de cet article, notamment par photocopie, n'est autoris que dans les limites des conditions gales d'utilisation du site ou, le cas hnt, des conditions gales de la licence souscrite par votre ablissement. Toute autre reproduction ou reprentation, en tout ou partie, sous quelque forme et de quelque manie que ce soit, est interdite sauf accord prlable et rit de l'iteur, en dehors des cas prus par la lislation en vigueur en France. II est prisque son stockage dans une base de donns est alement interdit. 


\section{Claire Squires}

\section{L'ombre de l'enfant non né sur la grossesse suivante}

"Mon corps est une tombe ", dit une mère qui a perdu son enfant au cours de sa grossesse. "Le temps semble s'être arrêté depuis ma grossesse précédente ", remarque une autre femme enceinte peu de temps après une mort in utero. Constatant à quel point les futures mères, en parlant de leur grossesse actuelle, celle qui suit une mort in utero, donnent l'impression de parler de la précédente, des projets qu'elles avaient conçus pour l'autre enfant, nous avons voulu mieux connaître les effets de la mort in utero sur la grossesse suivante. Nous nous sommes interessées à la façon dont des parents bouleversés par une mort in utero antérieure accueillent un enfant conçu ultérieurement et comment s'instaure, dans ce contexte, la relation avec celui-ci. Il s'agit, en retour, de savoir comment l'enfant, au début de sa vie, induit un effet sur les parents, l'anticipation prénatale de l'enfant risquant d'être sidérée par la perte fœtale précédente. Mais, inversement, l'attente d'un autre enfant peut permettre la poursuite d'un travail psychique réparateur.

La proximité de la vie et de la mort, qu'on côtoie quotidiennement en maternité, surprend avec violence chaque clinicien qui y est confronté. Cette juxtaposition à la fois familière dans la littérature, les croyances, et énigmatique dans sa formulation, commence à prendre forme. Ne dit-on pas, en effet, « renaître de ses cendres » ? Le mythe égyptien selon Hérodote raconte que cet oiseau fabuleux, unique en son espèce vivait plusieurs siècles, et, brûlé, reprenait vie. Quel est donc le sens de cette légende?

Claire Squires, psychiatre, psychanalyste, maître de conférences à Paris VII, Institut Claparède, Neuilly (92).clairesquires@noos.fr

Nous remercions les professeurs Émile Papiernik et Dominique Cabrol, chefs de service de la maternité de Port-Royal-Cochin (Paris). 
LES ÉTUDES SUR LE DEUIL PÉRINATAL ET LES ENFANTS DE REMPLACEMENT

Il existe peu d'études sur la grossesse suivant une mort in utero, en particulier aucune recherche clinique n'a été menée sur les relations précoces mère-enfant. Le travail le plus riche cliniquement dont nous disposons a un titre évocateur : «La grossesse suivant une mort in utero, la parentalité face à l'incertitude » de S. Philipps (1985). Ce travail rétrospectif est issu des interviews de quinze couples de parents entre trois et cinq ans après la naissance d'un enfant suivant une mort in utero. Il est centré sur les stress spécifiques lors de la grossesse suivante et sur l'adaptation des parents face à cette expérience. Le terme anglo-saxon de coping est difficile à traduire en français : il s'agit de faire face, faire avec, se comporter.

En général, les mères sont plus anxieuses et souhaitent planifier une grossesse plus tôt que les pères. Si la grossesse suivante tarde, les couples craignent une infertilité chronique. Les pères ont tendance à protéger leurs femmes sans exprimer leurs propres craintes de peur d'augmenter l'anxiété de leurs épouses. Le plaisir de la maternité s'efface devant une nette tendance à l'auto-protection. Les dates anniversaires commémorant la mort de l'enfant précédent sont angoissantes. Après ces dates, l'anxiété diminue temporairement... Elles ont conscience que leurs craintes sont irrationnelles mais ne peuvent s'empêcher d'être inquiètes. L'anxiété porte sur tout le déroulement de la grossesse mais aussi au moment de la conception : l'annonce de la grossesse est perçue avec une certaine ambivalence. Leurs craintes sont réactivées lors des visites, des examens, de l'approche de l'accouchement. Cette étude suggère donc l'existence d'une anxiété spécifique au cours de la grossesse suivante mais ne précise pas les effets de l'anxiété sur la relation mère-enfant.

La persistance d'idées liées à l'enfant précédent, d'auto-reproches, de culpabilité, de colère projetée sur l'entourage ou les médecins rend la notion de deuil particulièrement discutable. En effet, si classiquement, on différencie la dépression du deuil par l'existence de pensées négatives retournées contre soi, le deuil en cas de mort in utero est atypique parce qu'il est toujours marqué par une forte culpabilité. Cette altération de l'estime de soi soulève une insécurité et un sentiment d'inadéquation du rôle de la femme et de la mère.

Après l'accouchement, les réactions des parents diffèrent : les pères se sentent soulagés de voir l'enfant sain et en vie ; les mères, en revanche, ont plus besoin de réassurance à propos de la santé de l'enfant. Parfois, elles ressentent une difficulté à s'y attacher, une tendance à trop protéger l'enfant, une anxiété persistante dans quelques cas. La présence de l'enfant vivant ravive souvent les pensées à propos de l'enfant mort. L'élation et l'excitation, 
le sentiment de deuil sont secondaires ; ces sentiments contradictoires troublent les parents qui occasionnellement tentent de comparer les deux enfants (Phipps, 1985).

Une autre étude sur le deuil périnatal se rapportant aux grossesses suivant une interruption médicale de grossesse montre l'impact de la perte d'un enfant. Les auteurs suédois ont comparé ces vingt-sept mères à vingt-neuf mères sans antécédents ayant donné naissance à un enfant suivant normal (Hunfeld et al., 1997). L'évaluation a été réalisée à l'aide d'un entretien semistructuré, d'un questionnaire d'anxiété (Spielberg State trait Inventory), d'un questionnaire de dépression et d'anxiété (general health questionnaire), d'un questionnaire de dépression post-natale (EPDS de Cox) ainsi que d'un questionnaire portant sur l'adaptation de la mère à son enfant (Broussard) où la mère évalue les différences entre un bébé normal et son propre enfant. Les mères ayant connu une perte d'enfant vivent leur grossesse de façon plus négative. Elles sont plus anxieuses, plus déprimées et plus inquiètes. Un mois après l'accouchement, elles estiment que leurs enfants ont plus de problèmes de sommeil, de pleurs, d'alimentation et de comportement qu'un bébé normal.

Ainsi, il apparaîtrait que les modèles développementaux classiques de la grossesse normale ne sont pas adaptés aux situations de perte périnatale. Deux phénomènes apparaissent, en effet, spécifiques : tout d'abord, la nécessité de se protéger et de contrôler la situation par une vigilance excessive. Le second phénomène observé consiste en une sensibilisation aux éventuelles issues négatives de la grossesse (comme la sensibilisation allergique).

Le délai nécessaire pour concevoir un autre enfant après la mort périnatale suscite des discussions :

- d'une part, les auteurs craignent que les parents ne s'accordent pas assez de temps pour commencer une autre grossesse. Bowlby dans Attachement et perte (1984) suggère d'attendre un an ;

- d'autre part, $d$ 'autres auteurs considèrent qu' une grossesse suivante contribue à alléger les conséquences du deuil (Jensen et Zahourek, 1972 ; Wolf, 1970).

Ces deux attitudes, en apparence opposées, témoignent de l'embarras des cliniciens. D'ailleurs, les conseils sont toujours mal reçus quoi qu'il arrive. La perte d'un enfant au cours de la grossesse constitue un événement non négligeable ; les réactions à cette mort in utero grèvent toujours le déroulement de la grossesse suivante quel que soit le délai à concevoir.

Lewis (1979) expose l'hypothèse qu'une grossesse trop rapprochée inhiberait le deuil et pourrait conduire à une idéalisation, voire un rejet de l'enfant, et à une maltraitance. 
À la suite d'une perte périnatale, les taux de conception augmentent en raison d'une sorte de compulsion à remplacer l'enfant (Vogel et Knox, 1975 ; Park et al., 1979). Par exemple, une étude a montré que les mères d'enfants morts d'une malformation cardiaque congénitale concevaient bien plus vite que les mères d'enfants survivant à une malformation cardiaque ou bien que les mères d'enfants survivant à une malformation cardiaque ou bien que les mères d'enfants en bonne santé (Rubin et Ferencz, 1985). En revanche, cette étude n'a pas montré comment la relation avec l'enfant suivant était affectée.

Si dans certaines études, le fait de devenir rapidement enceinte peut prolonger le deuil et majore la difficulté à s'attacher à l'enfant (Rowe et al., 1978) ; d'autres auteurs ne voient pas de différence concernant le deuil par rapport au délai à concevoir (Laroche et al., 1984). Jensen et Zahourek (1972) et Wolf et al. (1970) considèrent même la grossesse suivante comme un indice de résolution du deuil.

Les différentes études concernant le délai utile à surmonter la mort in utero et concevoir un autre enfant sont donc contradictoires. Différencier les mères pour qui la grossesse suivante aide à résoudre la problématique de deuil et les mères qui l'évitent s'avère difficile (Zeanah, 1989).

Les travaux sur la mort fœtale in utero portent principalement sur les conséquences immédiates du deuil et non sur les conséquences durables du deuil. Les facteurs de vulnérabilité accrue pour les pertes périnatales sont les antécédents d'instabilité émotionnelle (Wolf, Nelson, Schiller, 1970), les antécédents psychiatriques repérés (Toedter et Lasker, 1991), la suppression initiale des sentiments de deuil (Cullberg, 1972), la présence d'un jumeau survivant ou une nouvelle grossesse survenant moins de cinq mois après le deuil (Rowe, Clyman, Green, Mikkleson et al., 1978). Les auteurs cherchent à définir les facteurs favorisant un deuil pathologique mais d'une façon générale, elles sont peu concluantes.

On ne peut manquer d'être questionné par la pertinence d'établir des scores de souffrance périnatale portant sur les représentations conscientes et non sur la clinique de manifestations du deuil, notamment sur ses représentations inconscientes. Il nous semble que les études ne portent pas sur les mécanismes psychiques en jeu dans cette perte d'un fœetus dont il est loin d'aller de soi qu'elle entraîne un « deuil ».

De nombreux travaux ont montré l'impact irréversible de certaines expériences précoces, source de maltraitance et de séquelles neuropsychologiques et développementales profondes. Ainsi l'enfant, dès son plus jeune âge, est sensible aux troubles de la relation engagée avec ses parents (Fonagy, 1996). Cain et Cain (1964) et Poznanski (1972) notent que l'enfant suivant une perte pourrait être considéré comme le remplaçant de l'enfant perdu, jamais perçu comme individué. De façon générale, il existe peu de publications à 
propos des enfants de remplacement; il s'agit généralement d'enfants rencontrés par les psychiatres dans l'après-coup de la perte, le caractère de remplacement étant reconstruit à partir de la biographie d'un enfant suivi en thérapie pour des symptômes divers. Les réactions parentales consistent en un besoin d'être près de l'enfant, une protection excessive, des attitudes restrictives.

Cain A.C. et Cain B.S. (1964) définissent l'enfant de remplacement comme les enfants ayant été conçus peu de temps après la mort d'un autre enfant : " L'intention spécifique des parents qui ont cet enfant étant qu'il remplace ou se substitue à leur enfant qui est mort. »

Les auteurs décrivent les mères de ces enfants comme coupables, dépressives, phobiques ou compulsives avant la mort de l'enfant dans leur famille. Une préoccupation exagérée à propos des maladies et des accidents caractérise ces parents.

Dans l'article de Poznananski (1972), l'enfant de remplacement se réfère à un enfant se substituant à un enfant mort. parfois, un enfant est conçu à cet effet ; ailleurs, un autre enfant, en général plus jeune, est assigné à cette place. L'idéalisation serait une défense pour éviter des sentiments de colère envers une personne morte. Or, ces sentiments agressifs sont dans une certaine mesure une réaction normale. Mais lorsque cette idéalisation atteint une telle extrémité, elle est préjudiciable au développement de l'enfant.

Le deuil nécessite la reconnaissance de la perte de l'enfant. Habituellement, il se manifeste par de fréquents accès de colère disproportionnés et de désespoir qu'un tel malheur soit survenu et des souvenirs vivaces de l'enfant perdu durant une longue période jusqu'à des mois ou des années après. Graduellement, les parents deviennent capables de se détacher de leur investissement émotionnel et de réinvestir leur énergie vers d'autres relations. Si les parents ne sont pas prêts à le faire, ils ne peuvent pas élever un nouvel enfant dans un environnement émotionnel favorable. Remplacer un enfant par un autre entraîne les parents à un certain déni de la mort du premier enfant. Le remplacement de l'enfant agit alors comme une barrière à la reconnaissance de la mort de l'enfant parce que l'enfant réel existe comme un substitut. Ainsi, les premières étapes du deuil sont prématurément interrompues et le processus de deuil continue indéfiniment avec l'enfant de remplacement agissant comme le vecteur du deuil parental.

Green et Solnit (1964) décrivent le syndrome de l'enfant vulnérable se séparant, infantilisé et objet de préoccupations concernant sa santé. Ce syndrome survient lors d'événements menaçant la vie de l'enfant mais aussi en cas de préoccupations excessives durant la grossesse ou de deuils non résolus prédisposants. 
Michel Hanus (1982) parle de l'enfant de remplacement comme la résultante d'un choix prématuré d'un nouvel objet, comme la modalité défensive d'un deuil non fait pour en occulter la perte. Ainsi, les affects douloureux d'affliction sont évités. S'agissant d'un enfant, celui-ci occupe toujours la place d'un remplaçant narcissique sous la forme de la représentation inconsciente de leur narcissisme primaire, l'infans mirabilis (Serge Leclaire, 1975).

Or l'enfant, pour s'individualiser en tant que personne, doit renoncer progressivement à cet investissement narcissique ; s'il meurt, le narcissisme parental est profondément blessé, ce qui rend le deuil impossible et nécessite son remplacement. L'enfant remplaçant peut venir aussi remplacer un enfant vivant mais malade. Il revêt un rôle réparateur pour ses parents, ou bien il est conçu alors que l'autre enfant est voué à la mort. M. Hanus souligne le statut de substitut œdipien que revêt fréquemment l'enfant mort. De ce fait, son sexe a une importance diverse pour chacun des parents. La culpabilité intense, « le sentiment honteux et insupportable d'avoir en soi quelque chose de dangereux, de funeste, de mortifère, une force maléfique comme démoniaque, éventuellement associée à l'idée d'un destin maléfique " éprouvée par les parents s'expliquerait par la blessure narcissique liée à la mort de l'enfant.

Dans un autre travail plus récent sur les deuils, Hanus (1995) ajoute que l'enfant de remplacement est un objet surmoïque interdit. Ainsi, l'enfant mort devient pour les parents un persécuteur potentiellement puissant. L'enfant suivant aussi aura la charge d'assumer à la fois sa différence avec son aîné, et sa ressemblance inévitable avec lui, parce que pour les parents, il sera toujours le même, toujours le remplaçant. Il devra affronter à la fois l'idéalisation et parfois le rejet lié aux affects négatifs que suscite la disparition du premier enfant. L'absence de travail de deuil de la part des parents fragilise le destin de l'enfant suivant dans la mesure où il devra lui-même l'effectuer : "Le deuil non fait par les parents constitue un point de fixation dans leur psyché où se jouent et leur agressivité méconnue pour un enfant mort et la culpabilité qui en découle, deuil repris par l'enfant de remplacement qui ne peut qu'en démêler les fils s'il veut libérer son identité propre et son autonomie. »

Les textes se rapportant à l'enfant de remplacement portent généralement sur des enfants ayant vécu et pouvant avoir été reconnus par les parents avec leurs caractéristiques propres. Un enfant mort in utero n'est pas encore aussi individualisé. Toutefois, M. Hanus souligne qu'un enfant très jeune est massivement investi du narcissisme parental.

L'enfant suivant est aussi un enfant réparateur. Cette hypothèse présente tout autant que la précédente, l'intérêt de décrire les mouvements inconscients parentaux. D'ailleurs, ces différentes hypothèses ne me paraissent nul- 
lement exclusives l'une de l'autre puisque l'enfant suivant sera peut-être réparateur du narcissisme parental, d'autant plus que la culpabilité liée au ressentiment à l'égard de l'enfant mort aura été expurgée. Nous détenons ici une piste particulièrement intéressante pour notre recherche.

À travers une analyse bibliographique qui porte essentiellement sur des cas d'enfants plus âgés, il est difficile de savoir comment la circonstance de la mort de l'enfant intervient sur le développement de l'enfant suivant. Les parents reconnaissent volontiers que des images d'enfant mort se superposent à celles de l'enfant à venir ; mais peut-on véritablement parler d'enfant de remplacement? L'enfant suivant lors de la grossesse mais aussi plus tard, en effet, sera l'objet de craintes et de soins redoublés ; ses parents pourront refouler, dénier, cliver des affects douloureux. D'autant plus peut-être que la première grossesse avait été idéalisée et que cet enfant mort reste dans le psychisme parental à une place de l'enfant parfait persécuteur du narcissisme primaire parental. On ne peut savoir ce qu'il adviendra du destin de cette perte chez l'enfant suivant; toutefois, notre recherche nous laisse penser que les autres enfants de la fratrie sont extrêmement sensibles à la présence d'un autre inconnu, vraisemblablement perfectible.

Le secret portant sur l'enfant mort antérieurement est-il lourd à porter ? Mais, doit-on faire part à l'enfant jeune des deuils qu'on peine à évoquer comme parent?

Si les études bibliographiques montrent toutes l'existence de réactions affectives marquées chez les parents lors d'une mort fœtale, la façon dont elles s'organisent est moins claire. Il est étonnant de lire que la plupart des auteurs parlent d'un deuil, comme un comportement de mauvaise adaptation sociale, voire d'état psychique négatif, sans questionner même ce que les parents ont perdu à travers l'enfant. En d'autres termes, peut-on faire le deuil de ce qu'on ne connaît pas, de quelqu'un avec qui on n'a pas eu d'échanges? Les processus en jeu dans la résolution d'une perte fotale doivent être donc mieux explicités.

Charles Zeanah, un professeur de pédopsychiatrie, dans une revue générale de la littérature sur le deuil périnatal (1995) remarque : "Peut-être, le problème majeur de toutes les recherches sur les conséquences d'une perte périnatale réside dans l'appréciation du champ étroit de la symptomatologie affective au détriment d'une compréhension des processus de deuil... Beaucoup de choses doivent être clarifiées concernant l'adaptation des parents, notamment les différences individuelles des symptômes de deuil, les différences culturelles, les différents types de pertes périnatales et les relations entre la perte et les enfants nés après la mort in utero. »

Le problème soulevé par cet auteur guide notre réflexion car il s'agit bien de savoir comment apprécier la nature de la perte lors d'une mort in 
utero. En d'autres termes, doit-on mener une exploration comportementale ou bien une investigation de type psychanalytique des processus à la fois conscients et inconscients à propos du deuil périnatal ?

Pour cette étude, nous avons rencontré une cinquantaine de couples qui attendaient un enfant puîné dans une maternité (Port-Royal) en travaillant en étroite collaboration avec un obstétricien sensibilisé à ce sujet, le professeur Émile Papiernik. Nous exposerons ici quelques aspects de cette clinique singulière d'une naissance dans un contexte de deuil puis nous discuterons le statut de ce deuil en ouvrant quelques hypothèses métapsychologiques sur la place de l'enfant mort et le statut de la violence par rapport au maternel et au féminin.

\section{LA GROSSESSE SUIVANTE FIGÉE PAR LA PERTE}

Non exceptionnellement, les mères évoquent le souvenir de la mise au monde de leur enfant comme l'expérience la plus marquante mais aussi la plus effrayante de leur existence. Et particulièrement lorsqu'une menace réelle ou imaginaire a pesé sur leur vie à elle ou sur celle de leur enfant. Or, l'effroi lié à la surprise et à l'impréparation lors de l'annonce de la mort in utero, marque la réaction initiale. Plus tard, même longtemps après, des images apparaissent à propos de l'enfant mort, de façon imprévisible, ravivées lors de la grossesse suivante. Celle-ci, marquée par le traumatisme, est toujours revécue comme une grossesse à risque.

En effet, cette nouvelle conception apparaît figée par la perte ; lors des consultations, la tristesse, le manque d'élation contrastent avec les grossesses habituelles. Le masque de la grossesse s'efface devant un visage immobile, inquiet. Des femmes habituellement coquettes ne se fardent plus, vêtues de couleurs sombres, dans des tenues peu recherchées. Un certain ralentissement, un émoussement des affects est visible. L'absence de joie s'accompagne d'une tension extrême à la crainte de la récidive. Une fois qu'elles se sentent un peu rassurées, l'atmosphère des consultations devient moins pesante, comme si elles reprenaient goût à la vie. D'un moment à l'autre, si quelque signe inattendu survient, l'anxiété est réactivée, les craintes réapparaissent.

Même si les mères souhaitent à tout prix enfanter à nouveau, l'annonce de la grossesse est souvent reçue sans plaisir, comme une éventualité, nécessaire mais préoccupante. L'idée d'une tâche à accomplir. Cette absence de ravissement s'accompagne d'emblée d'une vigilance exacerbée pouvant aller jusqu'au refus d'être enceintes. Cette inquiétude sur la viabilité du fœtus règne sur le déroulement de la grossesse, parfois plus intensément lors de la date anniversaire de la mort du bébé précédent. Lors de ces grossesses, les mouvements du fœtus n'éveillent pas comme dans les grossesses habituelles 
la vie imaginaire de la mère, les sensations auto-érotiques, les projections se rapportant habituellement au fotus, en dehors de la levée de l'angoisse. Une mère, par exemple, avait demandé qu'on enregistre en continu jour et nuit la mobilité de l'enfant qu'elle portait à l'aide d'un appareil médical afin d'être sûre que celui-ci ne puisse s'arrêter de bouger sans qu'on s'en aperçoive.

Un travail psychique de différenciation peut s'engager lorsqu'elles perçoivent des différences dans le déroulement des deux grossesses. Les mères disent, par exemple, que cet enfant-là bouge beaucoup alors que le précédent apparaissait plus calme.

\section{L'EXPRESSION SOMATIQUE DE LA DOULEUR PSYCHIQUE}

Les femmes nous paraissent souvent minimiser leurs craintes voire nier leurs angoisses de mort auprès de l'obstétricien à qui elles font part de nombreux symptômes somatiques. Près de la moitié d'entre elles seront hospitalisées pour les motifs les plus divers. Elles accouchent souvent à leur demande ou à celle du médecin par accouchement provoqué ou par césarienne. Les représentations pénibles sont repoussées ou bien refoulées. De nombreuses configurations psychopathologiques (plaintes hypocondriaques, mélancolie, symptômes phobiques ou manifestations hystériques) peuvent survenir dont le statut reste incertain selon les cas. Toutefois, les entretiens laissent apparaître les images ayant trait à la mort in utero. L'enfant en train de grandir est occulté aux dépens des souvenirs de la grossesse précédente, réactivés par le vécu actuel. La plainte phobique résulte du déplacement d'autres pensées, plus secrètes, ayant peut-être trait à l'idée de la mise à mort de l'enfant.

Mme I.T. est une jeune femme très préoccupée, ressassant l'idée morbide qu'elle est peut-être responsable de la mort de son enfant. Depuis cet épisode, elle se sent inutile, ne sait pas comment occuper son temps libre. Elle craint de se retrouver dans un lieu sombre, confiné, hors de chez elle et a dû renoncer aux week-ends d'évasion avec son conjoint. Elle ressent alors une grande angoisse. Elle pense que cette angoisse est liée à la peur que son enfant mort ait pu souffrir dans son ventre et qu'il ait pu lui aussi ressentir cet étouffement. Lors des entretiens suivants, cette angoisse s'est atténuée, en dépit du fait, dit-elle, qu'elle se trouve proche du terme de la mort de cet enfant.

La culpabilité n'est pas toujours aussi clairement exprimée de la sorte ; on pourrait y voir l'effet de la nécessité pour des parents de s'identifier à leur enfant non-né et de pouvoir exprimer la souffrance de l'enfant en eux. Les images ne sont pas des images de fœtus mais plutôt des reliquats imaginaires ou fantasmatiques au sujet de l'enfant. L'agonie de l'enfant est revécue 
comme une scène de mise à mort, la mort vécue, agie par la mère. La mère se sent l'agent de cette mise à mort. Comme si la puissance créatrice maternelle pouvait contenir, en germe, la destruction. Ces scénarios construits de la mort de l'enfant aident au processus de séparation, à atteindre le sentiment de culpabilité. Se séparer du mauvais objet pour que le narcissisme primaire soit restauré laisse la place au bon objet, l'enfant de la grossesse suivante. Pour renaître et réinvestir l'objet psychique, il faut se séparer des représentations morbides. L'objet perdu reste à perdre. Certaines femmes ont eu parfois l'impression que l'enfant bougeait plus vivement avant sa mort. " Peut-être aurait-on pu le sauver ? » D'autres femmes se sentent coupables de n'avoir pas ressenti qu'il ne bougeait plus. Se représenter la personne qu'on a connue, en rêver, faire revivre un être cher décédé, permet d'accepter la séparation. Pour un enfant mort in utero, le souvenir est plus ténu. Pour cette raison, voir le corps de l'enfant mort aiderait au processus de détachement. Toutefois, même pour les mères qui ont vu l'enfant, cela n'empêche pas les scénarios fantasmatiques.

Lorsque les restes mnésiques n'apparaissent pas sous forme de représentations de mots ou d'images, le corps devient le théâtre du retour des affects refoulés. En dehors de ces symptômes phobiques, les mères ont plus souvent tendance à souffrir de maux divers évoquant des plaintes hypocondriaques plutôt centrées sur le corps maternel. Pierre Fédida postule dans Le site de l'étranger que certaines figures psychopathologiques évoquent la mise en échec de la pensée du cadavre. Notamment dans l'hypocondrie (1995).

Freud, dans Pour introduire au narcissisme, inclut l'hypocondrie dans les névroses actuelles à côté de la neurasthénie et de la névrose d'angoisse. "L'hypocondrie retire intérêt et libido - celle-ci avec une évidence particulière - des objets du monde extérieur et concentre les deux sur l'organe qui l'occupe. »

L'hypocondrie dépendrait pour Freud de la libido du moi alors que dans l'hystérie et la névrose obsessionnelle, elle dépendrait de la libido d'objet. L'angoisse hypocondriaque serait, de la part de la libido du moi, le pendant de l'angoisse névrotique. La description freudienne de l'hypocondrie et de la mélancolie ne sont pas sans ressemblances, supposant une perte narcissique, puisque le mélancolique retire aussi sa libido et son intérêt pour le concentrer sur le moi, en une identification du moi avec l'objet abandonné. L'hypocondrie, selon Bernard Brusset, chez des patients confrontés à une perte d'objet de nature narcissique, éviterait la mélancolie par la régression narcissique et auto-érotique (1995). Ainsi, lors d'une grossesse suivant une mort in utero, la séparation toujours nécessaire avec l'enfant mort suscite tantôt des symptômes physiques, tantôt une nostalgie, tantôt une anxiété diffuse. On retrouve ici les ressemblances et les différences entre l'hypocondrie, douleur 
corporelle, et le deuil, douleur morale nostalgique. Le fait que la douleur se manifeste sous forme de déplaisir et non d'angoisse tient à la force de l'investissement et de liaison. Toutefois, si la nature narcissique ou objectale des investissements rend compte de la douleur corporelle hypocondriaque ou du deuil de l'objet perdu, de nombreux points de cette question demeurent obscurs : «Il semble aller de soi que la séparation de l'objet soit douloureuse. Le problème se complique encore : quand la séparation de l'objet produit-elle de l'angoisse ? Quand produit-elle le deuil et quand produit-elle seulement de la douleur?»

La douleur de la séparation insupportable peut parfois être niée et ignorée. Les parents oscillent entre un vécu douloureux clivé et des angoisses archaïques difficilement exprimables. Le vécu du deuil est souvent maintenu secret, comme une pensée magique, permettant une satisfaction hallucinatoire, correspondant au mode de pensée de l'enfance, à la façon d'un processus primaire, d'un vécu de la première enfance.

\section{LE DOMMAGE NARCISSIQUE, SOURCE DE VULNÉRABILITÉ}

Les femmes ressentent souvent une impression de vide, d'effondrement, liés à la brutale vacuité utérine et au tarissement des possibilités d'allaitement. Ces sensations sont repoussées à distance de l'événement. Des perceptions intéroceptives, tactiles, kinesthésiques marquent les sensations érogènes liées au déroulement de la grossesse précédente. Certaines mères se rappellent avec nostalgie les sensations de cette grossesse-là.

Mme X. vient demander un suivi psychologique alors qu'elle s'apprête à tenter une nouvelle grossesse après avoir perdu trois enfants d'un hématome rétroplacentaire. Elle a parfois l'impression de percevoir les mouvements d'un enfant dans son ventre.

Les femmes évoquent indirectement et avec une certaine réticence ces sensations en rapport avec la présence de l'enfant précédent par peur d'une pensée magique. Lors de la grossesse suivante, l'accent est porté sur les sensations corporelles plutôt que sur la douleur psychique ainsi que nous l'avons montré. Ce ressenti pénible semble commémorer l'absence de l'enfant précédent sous forme de douleurs mal systématisées, de contractions utérines, de douleurs en rapport avec la sphère gastrique. L'investissement narcissique est prégnant aux dépens de l'investissement d'objet comme dans toute grossesse, mais plus particulièrement du fait de la mort in utero. Ce qui est perdu avec l'enfant étant difficilement représentable, la sensation corporelle est privilégiée et la plainte hypocondriaque permettrait une issue autre que la mélancolie que Freud pense liée à la perte d'un objet dont on ne mesure pas les conséquences. 
«Peut-être les assauts multiples contre l'amour-propre de la mère contribuent plus à sa dépression que la perte de son bébé comme objet distinct. Certaines manifestations communes après la perte $d^{\prime}$ une grossesse, particulièrement l'impression de vide intérieur, d'irréalité, d'entendre un bébé, peuvent être ancrées non seulement dans les sentiments dépressifs habituels et dans les résidus physiologiques de la grossesse, mais également dans une perception fragmentée du soi et dans des symptômes somatiques similaires à ceux qu'expriment les gens qui subissent un dommage narcissique ", constate Irving Leon (1992).

Marguerite Duras écrit la solitude d'une mère qui vient de perdre son enfant dont elle cherche le souffle de son existence. Elle exprime très précisément les sensations de vidage, de déplétion du corps et du sentiment de vide de l'âme par opposition au ressenti érotisé puerpéral. Elle décrit le double mouvement d'une mère comme "l'horreur d'un pareil amour ", obligée d'imaginer l'existence de ce premier enfant en présence du second par l'écho de son souffle. L'écriture capture parfaitement en image et en sensation l'étrange présence de l'absence, la vie qui s'échappe, la représentation qui vient de naître et déjà s'évapore. L'écriture poétique trace particulièrement bien les impressions, les traces sur le corps de la mère de l'éphémère existence de l'enfant. Ce bref texte est écrit pendant la guerre vraisemblablement mais publié plus tardivement (Sorcières, 1976 ; Outside, 1984). Duras a perdu, une heure après l'accouchement, l'enfant qu'elle a eu avec Robert Anthelme. Elle demande aux religieuses à voir l'enfant.

" Je voudrais l'avoir près de moi une heure. Il est à moi », ce qu'on lui refuse comme cela se pratiquait à ce moment-là. « [...] Allongée toujours sur le dos, face aux acacias. La peau de mon ventre me collait au dos tellement j'étais vide. L'enfant était sorti, nous n'étions plus ensemble. Il était mort d'une mort séparée. Il y avait une heure, un jour, huit jours ; mort à part, mort à une vie que nous avions vécue neuf mois ensemble et qu'il venait de quitter séparément. Mon ventre était retombé lourdement sur lui-même, un chiffon usé, une loque, un drap mortuaire, une dalle, une porte, un néant que ce ventre. Il avait porté cet enfant pourtant, et c'était dans la chaleur glaireuse et veloutée de sa chair que ce fruit marin avait poussé. Le jour l'avait tué. Il avait été frappé à mort par sa solitude dans l'espace. Les gens disaient : “Ce n'était pas si terrible à la naissance, il vaut mieux ça." Était-ce terrible ? Je le crois. Précisément, ça ; cette coïncidence entre sa venue au monde et sa mort. Rien, il ne me restait rien. Ce vide était terrible. Je n'avais pas eu d'enfant, même pendant une heure. Obligée de tout imaginer. Immobile, j'imaginais.

Celui qui est là maintenant et qui dort, celui-ci, tout à l'heure, a ri. Il a ri à une girafe qu'on venait de lui donner. Il a ri et ça a fait un bruit de rire. Il y avait du vent et une petite partie du bruit de ce rire m'est parvenue. Alors, 
j'ai relevé un peu la capote de sa voiture, je lui ai redonné sa girafe pour qu'il rie de nouveau et j'ai engouffré ma tête dans la capote pour capter tout le bruit de ce rire. Du rire de mon enfant. J'ai mis l'oreille contre le coquillage et j'ai entendu le bruit de la mère. L'idée que ce rire était dispersé dans le vent, c'était insupportable. Je l'ai pris. C'était moi qui l'ai eu. Parfois quand il bâille, je respire sa bouche, l'air de son bâillement. S'il meurt, j'aurais eu ce rire. Je sais que ça peut mourir. Je mesure toute l'horreur d'un pareil amour. »

Le ventre s'évide, se désarrondit, se fripe après l'évacuation de l'enfant. La solitude, la banalisation, l'absence d'humanité, le pouvoir religieux sont aussi le destin des mères qui ont perdu un enfant. L'imagination sauve du désespoir de la mort. L'enfant suivant dont elle recueille les premiers rires, les premiers soupirs, est marqué par le souvenir de la fragilité de la vie du premier. En présence du suivant, la mère hallucine les traces fugitives du premier enfant mort né. Le visage de son enfant qu'on n'a pas vu ne permet pas à la mère de se reconnaître comme mère dans les yeux de l'enfant, comme le dit Winnicott. Si les spécialistes de la petite enfance ont accordé très tôt à l'enfant la capacité de reconnaître un visage animé, c'est aussi du côté des parents que l'empreinte se forme très précocement.

Mettre au monde un enfant sans vie, perdre la vie du fait de la naissance est une menace toujours redoutée mais en même temps elle n'est pas figurable : l'enfant immortel de l'avant-naissance accède au statut de mortel lorsqu'il vient au monde. Ainsi, la mise à mort est peut-être fantasmée mais si elle advient dans la réalité, elle est difficilement représentable.

L'ambivalence inhérente au désir d'enfant, surtout la possibilité qu'en donnant la vie on devient créateur tout puissant et aussi capable d'engendrer la mort, que toute vie contient en germe la mort, tous ces sentiments contradictoires affleurent à la conscience mais sont difficilement mis au jour. Ils peuvent donc resurgir lors d'une grossesse ultérieure sous forme d'une angoisse diffuse et difficile à mettre en mots.

\section{L'ATTEINTE À LA FÉMINITÉ}

Dans la mesure où le fœtus fait physiquement partie de la mère, l'enfant à naître est donc ressenti d'abord comme une partie d'elle-même et plus tard comme une personne séparée, un être étrange et familier. L'attachement et les liens de la mère en l'enfant seront toujours imprégnés de ce narcissisme. De nombreux psychanalystes ont souligné comment l'enfant que porte une mère réveille en elle le souvenir de l'enfant qu'elle fut avec sa propre mère. C'est grâce à ce sentiment de l'enfance qu'une mère pourra à la fois s'identifier comme mère et s'identifier à l'enfant, lorsqu'il sera né. Or, il est impossible de distinguer ce qui relève du féminin et du maternel tant ces deux aspects 
de la féminité sont intriqués. Winnicott parle de la maternité comme un état proche de la maladie normale. André Green évoque la folie maternelle primaire.

"Souvent », remarque Hélène Deutsch, le fotus " apparaît comme un enfant idéal, habituellement représentant la rêveuse elle-même, douée de ses meilleures qualités et de toutes celles qu'elle aimerait avoir» (1945).

L'enfant fait partie du corps de la mère, merveilleux et comblant d'où le sentiment de complétude au cours de la grossesse. Mais paradoxalement, il vient comme partie de son corps lui rappeler toute la problématique œdipienne et de la castration. On peut ainsi comprendre le sentiment de blessure narcissique qu'entraîne la perte de l'enfant. Ainsi, la dualité narcissisme et amour d'objet constituent un repère pour appréhender cette maladie maternelle normale. Sans développer ici plus avant les idées d'Hélène Deutsch, on constatera comment l'accès à la féminité et à la maternité comporte l'idée d'un deuil nécessaire : deuil lié au complexe de castration, séparation et retrouvailles avec la mère, échange d'un objet imaginaire de la mère au père. Mais est-ce vraiment un deuil terminé dans la mesure où les désirs archaïques de la petite fille et de la femme restent actifs tout au long de la vie ? Dans le cas d'une mort in utero, la femme est confrontée à la reviviscence des craintes archaïques liées à la castration, à la haine de la mère, au masochisme, au traumatisme de la séparation. Dans la mesure où l'enfant mort in utero risque fantasmatiquement de détruire ce qui la rend mère, le féminin en elle est aussi touché. On pourrait presque dire que le pénis qu'elle a désiré du père ne peut être un enfant et devient l'organe mort masculin en elle.

L'enfant mort semble parfois occuper inconsciemment la place d'un enfant d'un désir œdipien, l'enfant impossible. Freud dans La vie sexuelle et La féminité parle du désir infantile de la petite fille d'obtenir un jour le pénis du père qui devient à l'âge adulte celui d'obtenir un enfant du père, $d^{\prime}$ 'où le terme d'enfant œdipien ou d'ersatz du désir du pénis. L'enfant mort dans les représentations maternelles est en effet souvent l'enfant inscrit dans la filiation en place du phallus. Le fait qu'il disparaisse semble confirmer son statut incestueux impossible ce qui a pour conséquence l'émergence d'une culpabilité d'autant plus vive chez la mère. Pour le père, l'opération est psychiquement aussi lourde. Pour l'enfant suivant aussi, l'enfant mort sera un représentant des imagos parentales.

\section{LES VEUUX DE MORT}

Dans le cas rapporté ici d'un couple récemment formé, la grossesse est vécue dans la précipitation, comme si la mère ne se sentait pas prête à 
accueillir son enfant. La mort de l'enfant, en réalité, peu investi, survient peut-être comme un soulagement inavouable. Une infirmière se demandait si cela peut aider à en faire le deuil. Encore faudrait-il que la mère ne se sente pas trop coupable et qu'elle puisse faire le lien psychiquement entre l'absence $\mathrm{d}^{\prime}$ investissement de la grossesse et des vœux de mort, ici, inconscients.

Mme V. vient de perdre son enfant à trente-huit semaines d'aménorrhée de façon brutale à la suite d'un hématome rétroplacentaire qui a provoqué une hémorragie foudroyante. Dans la chambre, elle semble presque à côté de l'événement. En revanche, le père me demande d'emblée si c'est gravissime. Il a cru que son amie allait mourir en couches. Ils sont plongés dans l'obscurité bleutée de la chambre ; le store est baissé. Après un long temps d'étreinte avec son conjoint, elle semble retrouver ses forces, elle a envie de parler d'elle. Cette grossesse était imprévue ; elle a pensé faire une interruption de sa grossesse, puis elle a accepté son état mais sans plénitude.

Lors d'une seconde grossesse, Mme V. accepte les entretiens avec le psychothérapeute avec une certaine distance et sa mère est toujours présente aux consultations obstétricales. Elle accouche quelques semaines en avance d'un enfant fragile, irritable mais très présent dans le contact. À deux jours de l'accouchement, Mme V. est épuisée par un fort blues du post-partum. On n'en saura pas plus sur son enfant, Mme V. ne répond pas au courrier pour une rencontre à trois mois.

Il arrive que des parents soient entraînés dans des deuils successifs qu'ils ne peuvent élaborer, à l'origine de séparations, dont la résolution supposerait la perte d'une position toute-puissante, orientée par les pulsions de mort. Illustrant parfaitement une telle dynamique mortifère, Magaret Mitchell, dans Autant en emporte le vent, décline le thème des vœux de mort sur les enfants à naître ou déjà nés 1 . Le thème de la guerre pour le pouvoir dans le couple et de la mort des enfants constitue un motif romanesque puissant. Un couple se déchire, les épreuves de la guerre de Sécession sont nécessaires pour accéder à la maturité, la mort des enfants marque un tournant décisif dans les péripéties de la vie mouvementée de deux héros terribles. Scarlett O'Hara évite son nouveau mari, Rhett Butler, qu'elle ne peut se décider à aimer pour lui jeter à la figure qu'elle attend un enfant de lui.

"Vous savez bien que cet enfant est de vous, mais je n'en veux pas plus de vous. Aucune femme ne voudrait avoir un enfant de vous. Je voudrais. Oh! mon Dieu, je voudrais qu'il soit de n'importe qui, mais pas de vous! » Scarlett, à cette cinglante annonce, goûtant la colère qu'elle provoque, voit dans le visage de son mari quelque chose qu'elle ne peut analyser. « Ne vous 
inquiétez pas! fit-il en pivotant sur ses talons. Vous aurez peut-être une fausse couche. » " Pendant un moment, la tête lui tourna, elle songea à ce qu'était la maternité, avec ses nausées, son attente monotone, ses tailles épaissies, ses heures de souffrances, toutes ces choses dont un homme ne pouvait se rendre compte. Et Rhett osait plaisanter. Il n'y avait que la vue du sang sur son visage sombre pour calmer sa douleur. »

\section{LA DISQUALIFICATION MATERNELLE}

L'hypothèse initiale d'une préoccupation renforcée des parents lors d'une grossesse suivant une mort in utero s'est révélée juste dans de nombreux cas. On peut donc parler d'une certaine lenteur de la mise en place des liens, une sorte d'adaptation réciproque difficile, due surtout aux troubles narcissiques des mères. Les mères que nous avons rencontrées doutaient être capables d'assurer la première fonction vitale, de préserver l'existence de l'enfant. La mort in utero précédente leur donnait un sentiment de disqualification, de ne pas savoir être-mère. Elles recherchaient chez des proches ou chez le personnel soignant une présence maternelle requalifiante. Didier Houzel parle de la recherche d'une "imago tutélaire ", notamment chez les mères d'enfants autistes et psychotiques (1995).

"J'appelle ainsi le fantasme d'être incompétente en tant que mère, de ne pas savoir trouver les solutions qui conviennent et qu'une autre, sans doute aurait trouvées. Tout se passe comme si ces mères se sentaient mises en tutelle par une imago maternelle qui leur dénierait la possibilité et le droit d'être mère à leur tour, d'où le nom d" "imago tutélaire" que j' ai donné à cette instance surmoïque. »

Les mères, marquées par la mort in utero souffrent de l'abandon de la mère intérieure capable d'une présence continue, celle qui console des pertes, une mère éternelle qui ne disparaîtrait pas.

Une mère, Mme G. non suivie pendant sa grossesse, que j'avais rencontrée pour l'examen de Brazelton, m'avait beaucoup surprise en me disant : "J'aimerais bien que vous veniez chaque mois pour voir comment mon fils se développera jusqu'à l'âge de 20 ans. »

PARFOIS LORS DES PREMIÈRES SEMAINES SE POURSUIT

ENCORE L'ÉLABORATION PSYCHIQUE DE LA PERTE DE L'ENFANT MORT

À l'accouchement, dans les premiers jours, la présence de l'enfant peut permettre dans certains cas la poursuite du travail psychique de la mère à propos des pertes et des deuils. Dans d'autres situations, la demande de la 
mère semble s'évaporer comme si elle ne pouvait plus parler d'elle lorsque l'enfant est là.

Mme G. tient sa fille Céline dans ses bras qui tète le sein avec ardeur et me dit : «Il y a quelque chose dont j'aimerais vous parler : je ne me sens pas très câline avec ma fille, moins que pour Éric. » Éric a 5 ans, Mme G. a perdu deux enfants prématurément au Brésil, puis a fait quatre fausses couches. «J'ai eu envie, parfois, de jeter mon enfant par la fenêtre et j'ai éprouvé cela pour ma fille depuis que je suis rentrée à la maison. Ça dure juste quelques instants. Je me demande pourquoi. » L'évocation de sa propre agressivité semble la surprendre et lui permet d'associer sur sa famille. Sa mère vient d'arriver du Brésil et s'active dans la cuisine. Mme G. a perdu son père quand elle avait deux ans et $\mathrm{m}^{\prime}$ avait dit que pour elle chaque grossesse était associée à des morts de ses proches. Son frère homosexuel est mort du sida pendant la grossesse d'Éric et elle a perdu la grand-mère de son mari durant la grossesse de Céline. Éric est un enfant éveillé dont on dit qu'il ressemble au frère de Mme G. «C'est vrai, dit-elle, ma mère nous disait toujours : "Vois ton frère travaille bien à l'école." J'étais tellement bloquée avec les études ! » Mme G. durant toute sa grossesse avait très peur de ne pouvoir aimer à la fois deux enfants.

La grossesse à l'ombre de l'enfant mort-né conduit à considérer un travail psychique du négatif dans la procréation. Alors que la naissance est souvent idéalisée, sous l'angle d'un projet constructif, autour de la maternité et de la paternité, l'irruption de la mort confronte à des forces destructrices en jeu dans l'avènement $d$ 'un enfant : la toute-puissance menaçante pesant sur l'enfant, le transfert des âmes d'ancêtres, les rituels conjuratoires de la mort.

En tentant de cerner la spécificité de la perte en jeu dans la mort in utero et l'attente de l'enfant suivant, nous pensons avoir montré qu'elle suscite un deuil qui ne peut être un deuil. Les parents ne savent pas ce qu'ils ont perdu avec l'enfant mort et plus qu'ailleurs, cette mort est irreprésentable. Y a-t-il finalement un mort derrière l'enfant mort ? Y a-t-il un deuil lorsqu'un enfant naît mort ? Les traces de l'enfant mort aident les parents à reconnaître qu'il y a bien eu une vie puis une mort, mais elles ne suffisent pas à l'accepter.

Une distance s'établit entre le projet imaginaire de l'attente de l'enfant et la dénégation quant à l'image du corps. L'utérus devient le lieu de ces forces destructrices. La toute-puissance mortifère laisse penser que le psychisme pourrait entraîner la mort de l'enfant suivant. Y a-t-il eu un meurtre de l'enfant mort ? La symptomatologie des mères lors d'une grossesse suivante évoque bien ce questionnement à travers l'hypocondrie, la mélancolie, la phobie, la dépression. Elle conduit à se demander quel est le statut de cet enfant, surtout s'il s'agit d'un premier enfant, n'est-il pas l'enfant du père ? 
Cet enfant mort est-il le prix à payer de la culpabilité d'avoir un enfant qui ne soit pas un enfant du père?

Or, cette affliction, liée à la perte précédente, marque le déroulement de la grossesse et fige l'attente de l'enfant suivant. De plus, la menace d'une récidive de cette mort in utero, qui pèse sur le puîné, paralyse les capacités de figuration et d'anticipation des parents. Un travail psychique de différenciation des deux grossesses s'avère utile pour que l'enfant mort n'étende son ombre sur l'enfant suivant, à la naissance. Ce travail fait appel aux capacités de figuration psychique autour de la mort de l'enfant précédent : les circonstances de la grossesse précédente, de son arrêt, les sensations qui l'ont accompagnée, les projets qui l'entouraient, les fantasmes à propos du fœtus mort. L'expérience partagée avec un autre autour de ce décès d'un enfant attendu, d'un projet de vie, des autres deuils et des traumatismes passés, des processus identificatoires en jeu lors d'une grossesse facilitent le travail réparateur lors de la grossesse suivante. Évoquer le sens de cette mort in utero dans le déroulement d'une vie libère un peu les mères de leur potentialité destructrice agissante en tout un chacun. La grossesse suivante peut alors apparaître réparatrice pourvu qu'un soutien des capacités maternelles soit possible, dans le cadre d'une relation transférentielle.

On peut donc se demander si l'histoire de l'oiseau mythique invisible, toujours renaissant de ses cendres, n'apporte pas un éclairage sur notre sujet. L'enfant mort in utero n'aurait-il la place du phénix, une personne unique dans son genre, supérieur aux autres dans l'esprit de ses parents, en quelque sorte immortelle et dont les enfants suivants seront nécessairement tributaires?

BIBLIOGRAPHIE

BRUSSET, B. 1995. "Expérience du corps : l'hypocondrie », Revue internationale de psychopathologie, $\mathrm{n}^{\circ} 20$, Paris, PUF, p. 505 à 532.

CAIN, A.C. ; CAIN, B.S. 1964. "On replacing a child », J. Am. Acad. Child Psychiatry, 3, 443.

FonAGY, P. 1996. Communication orale, 6e Congrès de la WAIMH, Tempere (Finlande), 2528 juillet.

FreUd, S. 1914. «Zur Einfürung des Nazissmus », G.W., X, 138-170 ; tr. fr. dans La vie sexuelle, Paris, PUF, 1970, p. 81-105.

FREUD, S. 1933. "La féminité », Nouvelles conférences d'introduction à la psychanalyse, Paris, Gallimard, 1984, p. 167.

Green, M. ; Solnit, A.J. 1964. "Reactions to the threatened loss of the child ", Pediatrics, 57, 34-46.

Hanus, M. 1994-1995. Les deuils dans la vie, deuils et séparations chez l'adulte et chez l'enfant, Paris, Maloine, p. 285-291. 
Houzel, D. 1995. «Une application de la méthode d'observation des nourrissons : les traitements à domicile », dans S. Lebovici, R. Diatkine, M. Soulé (sous la direction de), Nouveau traité de psychiatrie de l'enfant et de l'adolescent, Paris, PUF, p. 519530 .

Hunfeld, J.A.M. ; TAselaAr-Kloos, A.K.G. ; Agterberg, G. ; Wladimiroff, J.M. ; PAsSCHIER, J. 1997. « Trait anxiety, negative emotions, and the mothers' adaptation to an infant born subsequent to late pregnancy loss : a case-control study ", Prenatal Diagnosis, vol. 17, 9, p. 843-851.

JENSEN, J.S. ; ZAHOUREK, R. 1972. "Depression in mothers who have lost a newborn », Rocky Mountain Medical Journal, 71, 61-63.

LEON, I. 1992. "The psychoanalytic conceptualization of perinatal loss ", American Journal of Psychiatry, 149, 1464-147.

LEWIS, E. 1979. " Mourning by the family after stillbirth or neonatal death », Arch. dis. Child., 54, 303-306.

PARK, C.B. ; HAN, H. ; CHOE, M.K. 1979. « The effect of infant death on subsequent fertility in Korea and the role of family planning ", AM. J. Public Health, 69, 557-565.

PHIPPS, S. 1985-1986. «Subsequent pregnancy after stillbirth : anticipatory parenthood in the face of uncertainity », Internal. J. Psychiatry in Medecine, vol. 15, 3, 243-263.

POZNANSKI, E.O. 1972. "The "replacement child" : a saga of unresolved parental grief », Behavioral Pediatrics, 81, 6, 1190-1193.

Rowe, J. ; Clyman, R. ; Green, C. ; Mikkelsen, C. ; Haight, J. ; Ataide, L. 1978. « Follow-up of families who experience a perinatal death », Pediatrics, 62, 166-170.

RubiN, J.D. ; FERENCZ, C. 1985. "Subsequent pregnancy in mothers of infants with congenital heart disease », Pediatrics, 76, 371-374.

TOEDTER, L.J. ; LASKER, J.N. 1991. "Acute versus chronic grief : the case of pregnancy loss », Amer. J. Orthopsychiat., 61, 4, 510-522.

Toedter, L.J. ; LASKer, J.N. ; AlHAdEFF, J.M. 1988. « The perinatal grief scale : development and initial validation », Amer. J. Orthopsychiat., 58, 3, 435-448.

Vogel, H.P. ; KNOX, E.G. 1975. « Reproductive patterns after stillbirth and early infant death », J. Biosoc. Sci., 7, 103-111.

ZEANAH, C. 1995. "Perinatal loss and infant mental health : an introduction », Infant Mental Health Journal, vol. 16, n² 2, été, 76-79.

Résumé

L'anticipation prénatale d'un enfant, dans le contexte d'une mort périnatale antérieure, risque d'être figée. Inversement l'attente d'un autre enfant peut permettre un travail psychique réparateur.

Le vécu psychologique des femmes enceintes à la suite d'une grossesse ayant abouti à une mort in utero a été recueilli au cours d'entretiens cliniques et les premiers liens avec les enfants suivants ont été observés.

Il s'agit donc d'une recherche clinique en psychopathologie et particulièrement d'un point de vue psychanalytique associée à une observation naturaliste et comparative des interactions parents-enfants, afin de prévenir l'impact de relations mal engagées avec l'enfant. 
Sur le plan métapsychologique, le modèle freudien du deuil est en question au-delà du domaine de la périnatalité : comment penser le deuil à propos de l'enfant mort avec qui la relation est fortement investie mais non représentable ? La mort in utero n'offrirait-elle pas une forme de figuration d'un objet narcissique de nature mélancolique? Quel est l'effet de la violence exercée sur le corps maternel sur le féminin ?

Mots-clés

Mort in utero, deuil périnatal, enfant de remplacement, mélancolie, hypocondrie.

THE LIMBO CHILD'S VEIL ON THE FOLLOWING PREGNANCY

In the context of a previous stilbirth, following baby's prenatal anticipation may be freezed. On the opposite, expecting another baby can restore parent's fellings. Clinical interviews were conducted with these women and first parents-baby interactions were observed. This is a psychopathological survey on a psychoanalytic line, in a preventive approach. On a metapsychological line, freudian mourning's model is questionned : how mourning can be thought about a dead baby with whom the relationship is highly invested but not represented ? Can stillbirth figurate a melancolic narcissic object? What is the impact of violence on a mother's body, on feminine?

Key words

Stillbirth, perinatal mourning, replacing child, melancolia, hypocondria. 